\title{
Markov Chains with Quasitoeplitz Transition Matrix
}

\author{
Alexander M. Dukhovny
}

\begin{abstract}
This paper investigates a class of Markov chains which are frequently encountered in various applications (e.g. queueing systems, dams and inventories) with feedback. Generating functions of transient and steady state probabilities are found by solving a special Riemann boundary value problem on the unit circle. A criterion of ergodicity is established.
\end{abstract}

Key words: Markov chains, quasitoeplitz matrix, transient probabilities, steady state probabilities, ergodicity criterion, Riemann boundary value problem.

AMS subject classification: $60 \mathrm{~J}, 60 \mathrm{~K} 25$.

\section{Introduction}

This paper investigates Markov chains with quasitoeplitz infinite transition matrices, whose elements, beginning from some $n$-th row and second column, depend on differences of indices. Such matrices offer a generalization of the so-called $\Delta_{m, n}\left(\Delta_{m, n}^{\prime}\right)$ matrix (see Abolnikov and Dukhovny [1]), where toeplitz rows have a zero part-left or right, respectively to $\Delta_{m, n}$ or $\Delta_{m, n}^{\prime}$.

Many stochastic processes encountered in applications have a transition matrix with a quasitoeplitz structure: embedded Markov chains describing the behavior of a queue in the queueing systems $M / G / 1$ and $G I / M / 1$ with various patterns of dependence on the queue length, the state of a storage in the inventory control theory, the level of a dam with level-depending release, etc. The existing methods for investigations of these problems rely heavily upon the $\Delta_{m, n}$ or $\Delta_{m, n}^{\prime}$ structure of transition matrix (see,

\footnotetext{
${ }^{0}$ Received: January 1989; Revised: March 1989
} 
e.g., Abolnikov and Dukhovny [1] or Neuts [7]), thus leaving aside many important applications. To overcome these restrictions we develop here a new approach, begun by Dukhovny [3], to find the generating functions (GF) of transient and steady state probabilities.

The essence of this approach is to find these GF's by the solution of some special Riemann boundary value problems on the unit circle in the complex plane. We refer to [5] for the method to resolve such problems and express necessary GF's through some unknown elements. Then we find these unknowns from special systems of linear equations and prove the uniqueness of their solution.

Complete consideration of ergodicity problems is given. A necessary and sufficient ergodicity condition is established. It is shown that some tractable "natural properties" of transition matrix (so-called conditions of "descent" to zero and "ascent" from zero, and existence of averages) ensure the chain's being irreducible and aperiodic.

\section{Definitions and Assumptions}

Definition 1. An infinite stochastic matrix $A=\left(a_{i j}\right)$ is called quasitoeplitz if there is an $n \geq 0$ and non-negative numbers $h_{j}, j=0, \pm 1, \pm 2, \ldots$, such that for any $i \geq n$

$$
a_{i j}= \begin{cases}h_{j-i} & j>0 \\ \sum_{l=-\infty}^{-i} h_{l} & j=0\end{cases}
$$

This implies, of course, that

$$
\sum_{-\infty}^{\infty} h_{j}=1
$$

We also assume that

$$
\forall i, i=1,2, \ldots, n-1, \exists j, 0 \leq j<i \text { such that } \begin{array}{lll}
\exists j<0 & \text { such that } & h_{j}>0 \\
& & a_{00}>0
\end{array}
$$

We call this group of assumptions "the conditions of descent." To put aside the trivial case in which states from 0 to $n-1$ are isolated from others, we introduce a condition of "ascent": there is some sequence $j_{0}=0$, $j_{1}, \ldots, j_{r}$ such that: 


$$
0 \leq j_{s}<n, \forall s<\tau ; j_{\tau} \geq n ; a_{j_{s-1} j_{s}}>0, s=1,2, \ldots, \tau .
$$

In other words, the condition of ascent enables the chain to exceed $n-1$, starting from zero.

The natural assumptions,

$$
\begin{aligned}
& \sum_{j=0}^{\infty} j a_{i j}<\infty, \quad i=0,1, \ldots, n-1, \text { and } \\
& \sum_{-\infty}^{\infty}|j| h_{j}<\infty
\end{aligned}
$$

express the existence of averages for the distributions involved. Conditions (3-8) take place in the overwhelming majority of applications, so from here on we refer to them as to "natural properties." To avoid unnecessary complications we also include here the assumption that the sequence $\left\{h_{j}\right\}$ is aperiodic, as is the case in most applications.

Let $\Gamma^{+}$be the interior of the unit circle $\Gamma:|z|=1$ of the complex plane, also $\Gamma^{-}:|z|>1, \bar{\Gamma}^{+}=\Gamma^{+} \cup \Gamma$, and $\bar{\Gamma}=\Gamma^{-} \cup \Gamma$.

Now we introduce some GF's essential in the forthcoming investigation:

$$
\begin{gathered}
A_{i}(z)=\sum_{j=0}^{\infty} a_{i j} z^{j}, \quad z \in \bar{\Gamma}^{+} ; \text {and } \\
H(z)=\sum_{-\infty}^{\infty} h_{j} z^{j}, \quad z \in \Gamma .
\end{gathered}
$$

Let $\left\{\xi_{k}\right\}$ be a Markov chain that has the transition matrix $A$. We denote the transient probabilities $p_{i}^{k}=P\left\{\xi_{k}=i\right\}$, the steady state probabilities $p_{i}=\lim _{k \rightarrow \infty} p_{i}^{k}$, and their GF's

$$
\begin{aligned}
& P^{k}(z)=\sum_{i=0}^{\infty} p_{i}^{k} z^{i}, \quad z \in \bar{\Gamma}^{+} \\
& P(z)=\sum_{i=0}^{\infty} p_{i} z^{i}, \quad z \in \bar{\Gamma}^{+}
\end{aligned}
$$

Let $x \in(0,1)$. Denote

$$
\begin{array}{r}
U_{i}(x)=\sum_{k=0}^{\infty} p_{i}^{k} x^{k}, \text { and } \\
W(x, z)=\sum_{i=0}^{\infty} \sum_{k=0}^{\infty} p_{i}^{k} x^{k} z^{i}=\sum_{i=0}^{\infty} U_{i}(x) z^{i}=\sum_{k=0}^{\infty} P^{k}(z) x^{k} .
\end{array}
$$


We define vectors

$$
\begin{aligned}
\mathbf{P}^{k} & =\left(p_{0}^{k}, p_{1}^{k}, \ldots\right) \\
\mathbf{P} & =\left(p_{0}, p_{1}, \ldots\right) \text { and } \\
\mathbf{U}(x) & =\left(U_{0}(x), U_{1}(x), \ldots\right)
\end{aligned}
$$

We define the operators $D^{i}, T^{+}, T^{-}$, and $T^{0}$ by their impact on the arbitrary Laurent series $a(z)=\sum_{-\infty}^{\infty} a_{j} z^{j}, z \in \Gamma$, belonging to the Banach space $L^{1}$ of the power series with absolutely summable coefficients.

$$
\begin{gathered}
D^{i} a(z)=a_{i} ; \\
T^{+} a(z)=\sum_{j=0}^{\infty} a_{j} z^{j}, \quad z \in \bar{\Gamma}^{+} ; \\
T^{-} a(z)=\sum_{j=-\infty}^{-1} a_{j} z^{j}, \quad z \in \bar{\Gamma} ; \text { and } \\
T^{0} a(z)=\sum_{j=-\infty}^{-1} a_{j}+\sum_{j=0}^{\infty} a_{j} z^{j}, \quad z \in \bar{\Gamma}^{+} .
\end{gathered}
$$

Note that if $a(z) \in L^{1}$, then functions $T^{+} a(z)$ and $T^{0} a(z)$ are also elements of $L^{1}$, so they are analytic in $\Gamma^{+}$and continuous on $\Gamma_{;} T^{-} a(z)$ belongs to $L^{1}$ and is analytic in $\Gamma^{-}$and continuous on $\Gamma$.

Now we may transform definition 1 to the following:

$$
A_{i}(z)=T^{0} z^{i} H(z) \quad i \geq n .
$$

\section{Transient State Probabilities}

The transitions of the chain $\left\{\xi_{k}\right\}$ are described by

$$
p_{j}^{k+1}=\sum_{i=0}^{\infty} p_{i}^{k} a_{i j}, \quad j=0,1,2, \ldots
$$

or, in vector form, by

$$
\mathbf{P}^{k+1}=\mathbf{P}^{k} A .
$$

Multiplying both sides by $x^{k+1}$ and summing over $k$ from 0 to $\infty$, we have

$$
\mathbf{U}(x)=x \mathbf{U}(x) A+\mathbf{P}^{0} .
$$

Then from (9) we obtain

$$
W(x, z)=x T^{0} W(x, z) H(z)+P^{0}(z)+\sum_{i=0}^{n-1} U_{i}(x)\left[x A_{i}(z)-x T^{0} z^{i} H(z)\right] .
$$


Now we consider (13) for $z \in \Gamma$. Let

$$
F^{-}(x, z)=T^{-} \sum_{i=n}^{\infty} U_{i}(x) z^{i} H(z),
$$

and rewrite (13) as follows:

$\sum_{i=n}^{\infty} U_{i}(x) z^{i}[1-x H(z)]=F^{-}(x, 1)-F^{-}(x, z)+P^{0}(z)+\sum_{i=0}^{n-1} U_{i}(x)\left[x A_{i}(z)-z^{i}\right]$.

Since, by definition, $W(x, z)$ is analytic in $\Gamma^{+}$and $F^{-}(x, z)$ is in $\Gamma^{-}$, (15) is (see, e.g., Gakhov [5]) a Riemann boundary value problem on $\Gamma$ in the class $L^{1}$.

Theorem 1. The GF $W(x, z)$ of the transient state probabilities of a Markov chain $\left\{\xi_{k}\right\}$ with a quasitoeplitz transition matrix at $n \geq 1$ is defined by the following formulas:

$$
\begin{aligned}
W(x, z)= & \sum_{i=0}^{n-1} U_{i}(x) z^{i} \\
& +z^{n} R^{+}(x, z) T^{+} z^{-n}\left[P^{0}(z)+x \sum_{i=0}^{n-1} U_{i}(x) A_{i}(z)\right] R^{-}(x, z) \\
& R^{ \pm}(x, z)=\exp \left(-T^{ \pm} \ln [1-x H(z)]\right) ;
\end{aligned}
$$

where the unknowns $U_{i}(x), i=0,1, \ldots, n-1$ are the unique solution of the system of linear equations

$$
\begin{aligned}
& \sum_{i=0}^{n-1} U_{i}(x) D^{j} T^{0}\left[z^{i}-x A_{i}(z)\right] \\
& \quad=D^{j} T^{0} P^{0}(z) R^{-}(x, z), \quad j=0,1, \ldots, n-1 .
\end{aligned}
$$

Before we prove Theorem 1, we consider the following lemma.

Lemma 1. If $a^{-}(z)=\sum_{-\infty}^{0} a_{j} z^{j} \in L^{1}$ then $T^{0} a^{-}(z)=a^{-}(1)$.

Proof. By definition of $T^{0}$

$$
T^{0} a^{-}(z)=\sum_{-\infty}^{-1} a_{i}+a_{0}=a^{-}(1)
$$

which proves Lemma 1.

Proof of Theorem 1. For $x \in(0,1),|x H(z)|<x<1$, so the functions $R^{ \pm}(x, z)$ and $R^{ \pm}(x, z)^{-1}$, defined by (17) are analytic in $\Gamma^{+}$and $\Gamma^{-}$ 
respectively, and their respective series expansions in powers of $z$ and $z^{-1}$ belong to $L^{1}$ (see, e.g., [5]).

Due to (17) we have an obvious equality on $\Gamma$ :

$$
[1-x H(z)]^{-1}=R^{+}(x, z) R^{-}(x, z) .
$$

Multiply both sides of (15) by $R^{-}(x, z)$ and apply $T^{0}$. We can see that $a^{-}(z)=\left[F^{-}(x, 1)-F^{-}(x, z)\right] R^{-}(x, z)$ satisfies the preconditions of Lemma 1 and $a^{-}(1)=0$. The operator $T^{0}$ doesn't change

$$
\sum_{i=n}^{\infty} U_{i}(x) z^{i} R^{+}(x, z)^{-1}
$$

as it is analytic in $\Gamma^{+}$and continuous on $\Gamma$, so we have

$$
\sum_{i=n}^{\infty} U_{i}(x) z^{i} R^{+}(x, z)^{-1}=T^{0}\left[P^{0}(z)+\sum_{i=0}^{n-1} U_{i}(x)\left(x A_{i}(z)-z^{i}\right)\right] R^{-}(x, z) .
$$

The left side here doesn't contain powers of $z$ with exponents less than $n$ in its expansion, so applying $D^{i}, i=0,1, \ldots, n-1$ to both sides of (20) we obtain (18). Using (18) we now easily transform (20) to (16) with the help of the obvious identity

$$
\sum_{i=n}^{\infty} D^{i} a(z)=z^{n} T^{+} z^{-n} a(z) .
$$

The system of linear equations (18) already has one solution constituted by actual transient state probabilities. Existence of other solutions would provide through (16) another solution of (12), say, $\hat{\mathbf{U}}(x)=\left(\hat{U}_{0}(x), \hat{U}_{1}(x), \ldots\right)$. Since functions $R^{ \pm}(x, z)$ belong to $L^{1}$, formula (16) provides the same property to $\hat{W}(x, z)$ related to $\hat{\mathrm{U}}(x)$. Thus, $\hat{\mathrm{U}}(x)$ and $\mathrm{U}(x)$ are different solutions of (12) in the space of rows with absolutely summable elements. This is impossible, because $A$ is stochastic and $|x|<1$, so the norm of $x A$ in this space is less than 1.

Hence the system (18) must have a unique solution, which completes the proof of Theorem 1.

Note that at $n=0$ we can obtain $W(x, z)$ from $(20)$ :

$$
W(x, z)=R^{+}(x, z) T^{0} P^{0}(z) R^{-}(x, z) .
$$




\section{Ergodicity}

Lemma 2. Let $\left\{\xi_{k}\right\}$ be a homogeneous Markov chain. If there is such a state $i_{0}$ that can be reached from any other and retained through one step then this chain is irreducible and aperiodic.

Proof. Suppose it is reducible and there are two classes of equivalent connectable states-then both must contain $i_{0}$ and therefore coincide. Suppose the chain is periodic with some period $d$, so the numbers of the steps in any loop must be divisible by $d$-then $d$ must be 1 , as there is a one-step loop from $i_{0}$ to $i_{0}$. These contradictions prove Lemma 2.

Theorem 2. With conditions of descent (3-5) a true Markov chain with quasitoeplitz transition matrix is irreducible and aperiodic.

Proof. Now it is obvious that conditions of descent provide the requirements of Lemma 2 in our case with zero being the state mentioned in this Lemma. (3) provides the possibility to go down in one step from the states exceeding $n-1,(4)$ does the same for the states from 1 to $n-1$. So starting from any positive $j$ the chain can reach zero in no more than $j-1$ steps. (5) provides the one-step "stopover" in zero, as is required by Lemma 2 . Theorem 2 is proved.

Theorem 3. Markov chain $\left\{\xi_{k}\right\}$ with quasitoeplitz transition matrix with natural properties is ergodic if and only if

$$
H^{\prime}(1)<0
$$

Proof. (Sufficiency.) Let (23) be true and let $x_{j}, j=0,1,2, \ldots$ Then from (7) and (8) $\forall i$

$$
\Delta_{i}=\sum_{j=0}^{\infty} a_{i j} x_{j}-x_{i}=\sum_{j=0}^{\infty} j a_{i j}-i<\infty
$$

Furthermore, $\forall i \geq n$ due to $(1)$,

$$
\Delta_{i}=H^{\prime}(1)-\sum_{j=-\infty}^{0} j h_{j-i}
$$

Due to (7), with $i \rightarrow \infty$

$$
\left|\sum_{j=-\infty}^{0} j h_{j-1}\right|=\sum_{j=-\infty}^{-i}(|j|-i) h_{j} \leq \sum_{j=-\infty}^{-i}|j| h_{j} \rightarrow 0 .
$$


Hence, going to the limit in (25) we obtain

$$
\lim _{i \rightarrow \infty} \Delta_{i}=H^{\prime}(1)<0 .
$$

With $\left\{\xi_{k}\right\}$ being irreducible and aperiodic due to conditions of descent and Theorem 2, (24) and (26), according to Foster's theorem [4], enforce the ergodicity of $\left\{\xi_{k}\right\}$.

(Necessity.) Suppose $\left\{\xi_{k}\right\}$ is ergodic. Then there are steady state probabilities, not all zeros, satisfying the equilibrium equations

$$
\begin{gathered}
p_{j}=\sum_{i=0}^{\infty} p_{i} a_{i j}, \quad j=0,1,2, \ldots \text { and } \\
\sum_{j=0}^{\infty} p_{j}=1
\end{gathered}
$$

Let us multiply both sides of (27) by $z^{j-n}$ and sum over $j$ from $n$ to $\infty$ :

$$
\sum_{j=n}^{\infty} p_{j} z^{j-n}=\sum_{i=0}^{n-1} p_{i} \sum_{j=n}^{\infty} a_{i j} z^{j-n}+\sum_{i=n}^{\infty} p_{i} \sum_{j=n}^{\infty} h_{j-i} z^{j-n} .
$$

For $z \in \Gamma$ the last equation reduces to

$$
\sum_{j=n}^{\infty} p_{j} z^{j-n}[1-H(z)]=\sum_{i=0}^{n-1} p_{i} \sum_{j=n}^{\infty} a_{i j} z^{j-n}-\sum_{i=n}^{\infty} p_{i} \sum_{j=n}^{\infty} h_{j-i} z^{j-n}
$$

At $z=1$ the left side and hence the right side also are zero, so we divide both sides by $z-1$ and take the limit with $z \rightarrow 1, z \in \Gamma$, with the help of L'Hôpital's rule:

$$
-H^{\prime}(1) \sum_{i=n}^{\infty} p_{i}=\sum_{i=0}^{n-1} p_{i} \sum_{j=n}^{\infty}(j-n) a_{i j}+\sum_{i=n}^{\infty} p_{i} \sum_{j=-\infty}^{n-1}(n-j) h_{j-i} \geq 0 .
$$

(Note that (8) ensures the uniform upper bound for the sums

$$
\sum_{j=-\infty}^{n-1}(n-j) h_{j-i}
$$

and thus provides the absolute convergence of the right side that enables us to use L'Hôpital's rule.)

Suppose that the right side of (29) is zero. As all of its components are nonnegative, they must also all be zero. With $\left\{\xi_{k}\right\}$ being ergodic, 
all $p_{i}$, corresponding to the only class of essential states, are positive. Due to conditions of descent this class contains zero, so $p_{0}>0$. Then due to the condition of ascent (6) then all the states in the sequence of possible ascent must be essential. The first part of the right side of (29) being zero enforces that the end of ascent can only be at $j=n$, so $p_{n}>0$. Hence by (4) there must be at least one nonzero term in the expression

$$
p_{n} \sum_{j=-\infty}^{n-1}(n-j) h_{j-n}=-p_{n} \sum_{j=-\infty}^{-1} j h_{j} .
$$

This contradiction proves that the right side of (29) must be positive, so (23) holds.

\section{$5 \quad$ Steady State Probabilities}

Theorem 4. The GF $P(z)$ of the steady state probabilities of the Markov chain $\left\{\xi_{k}\right\}$ with quasitoeplitz transition matrix with natural properties at $n>0$ is given by the formulas:

$$
\begin{array}{r}
P(z)=\sum_{i=0}^{n-1} p_{i} z^{i}+z^{n} R^{+}(z) T^{+}\left(z^{1-n}(z-1)^{-1} R^{-}(z) \sum_{i=0}^{n-1} p_{i}\left[A_{i}(z)-1\right]\right)(30) \\
R^{ \pm}(z)=\exp \left(-T^{ \pm} \ln z(z-1)^{-1}[1-H(z)]\right)(
\end{array}
$$

where the unknown probabilities $p_{i}, i=0,1, \ldots, n-1$, and $c$ form the only solution of the system of linear equations:

$$
\begin{aligned}
& \sum_{i=0}^{n-1} p_{i} D^{j} T^{0}\left[z(z-1)^{-1} R^{-}(z)\left(z^{i}-A_{i}(z)\right)\right]=c \delta_{j}^{0}, \\
& \quad c R^{+}(1)+\sum_{i=0}^{n-1} p_{i}\left[1-H^{\prime}(1)^{-1}\left(A_{i}^{\prime}(1)-i\right)\right]=1,1, \ldots, n-1 \\
& \left(\delta_{j}^{0} \text { is Kronecker's symbol }\right) .
\end{aligned}
$$

Proof. Rewrite the equilibrium equations (27) in the vector form

$$
\mathbf{P}=\mathbf{P} A
$$

and multiply both sides of this by the column-vector $\left(1, z, z^{2}, \ldots\right)$ from the right:

$$
P(z)=\sum_{i=0}^{\infty} p_{i} A_{i}(z)
$$


According to (8) this may be rewritten as

$$
P(z)=T^{0} P(z) H(z)+\sum_{i=0}^{n-1} p_{i}\left[A_{i}(z)-z^{i}\right]+\sum_{i=0}^{n-1} p_{i}\left[z^{i}-T^{0} z^{i} H(z)\right] .
$$

Let

$$
\Phi^{-}(z)=T^{-} \sum_{i=n}^{\infty} p_{i} z^{i} H(z)
$$

Hence $\Phi^{-}(z)$ is analytic in $\Gamma^{-}$. At $z \in \Gamma^{-}$we obtain

$$
\sum_{i=n}^{\infty} p_{i} z^{i}[1-H(z)]=\Phi^{-}(1)-\Phi^{-}(z)+\sum_{i=0}^{n-1} p_{i}\left[A_{i}(z)-z^{i}\right] .
$$

This is again a Riemann boundary value problem on $\Gamma$, but its coefficient $1-H(z)$ has roots on $\Gamma$; so to apply the same technique we have to make some prearrangements.

Let $\Delta_{\Gamma} \operatorname{Arg}(f(z))$ be the increment of argument of $f(z)$ when the argument $\varphi$ of $z=\exp (i \varphi)$ changes from 0 to $2 \pi$ and

$$
\operatorname{Ind}_{\Gamma} f(z)=(2 \pi)^{-1} \Delta_{\Gamma} \operatorname{Arg}(f(z)) .
$$

Lemma 3. If the sequence $\left\{h_{j}\right\}$ is aperiodic and $H^{\prime}(1)<0$ then

$$
\operatorname{Ind}_{\Gamma}[1-H(z)]\left(1-z^{-1}\right)^{-1}=0 .
$$

\section{Proof.}

Lemma 4. If the sequence $\left\{h_{j} \geq 0\right\}, j=0, \pm 1, \pm 2, \ldots$ is aperiodic and its sum is 1 , then on $\Gamma \backslash\{1\}$

$$
|H(z)|=\left|\sum_{-\infty}^{\infty} h_{j} z^{j}\right|<1 .
$$

Proof. Suppose there exist $z_{0} \in \Gamma \backslash\{1\}$ such that

$$
1=|H(z)|=\left|\sum_{-\infty}^{\infty} h_{j} z_{0}^{j}\right| \leq \sum_{-\infty}^{\infty} h_{j}=1
$$

We can see that the equality here is possible only if for every essential $j$ (i.e., $h_{j} \neq 0$ ) the numbers $h_{j} z_{0}^{j}$ are colinear. Denote by $j_{0}$ that essential index with the least absolute value. For every other essential $j, z_{0}^{j-j_{0}}$ must be 1 , hence 
all such numbers $j-j_{0}$ must have a common integer divider $m$ such that $z_{0}^{m}=1$. This contradicts the assumption of aperiodicity.

Now we return to the proof of Lemma 3 . Since $\lim _{z \rightarrow 1}[1-$ $H(z)]\left(1-z^{-1}\right)^{-1}=-H^{\prime}(1)>0$

$$
\lim _{\varphi \rightarrow \pm 0} \operatorname{Arg}\left[1-H\left(e^{i \varphi}\right)\right]=\lim _{\varphi \rightarrow \pm 0} \operatorname{Arg}\left[1-e^{-i \varphi}\right]= \pm \frac{\pi}{2}
$$

Due to Lemma 4 at $z \in \Gamma \backslash\{1\}, \operatorname{Re}[1-H(z)]>0$, so $-\pi / 2<$ $\operatorname{Arg}[1-H(z)]<\pi / 2$. Hence $\Delta_{\Gamma} \operatorname{Arg}[1-H(z)]=\pi=\Delta_{\Gamma} \operatorname{Arg}(1-$ $\left.z^{-1}\right)$, which yields (37) of Lemma 3 .

On the strength of $(37), \ln [1-H(z)]\left(1-z^{-1}\right)^{-1}$ belongs to $L^{1}$ (see e.g. [5]). Hence functions $R^{ \pm}(z)$ and $R^{ \pm}(z)^{-1}$ defined by (31) are also in $L^{1}$, so they are analytic in $\Gamma^{+}$and $\Gamma^{-}$respectively, and continuous on $\Gamma$ with no roots in these areas.

Now we multiply both sides of (36) with $\left(1-z^{-1}\right)^{-1} R^{-}(z)$ and use this obvious equality on $\Gamma$ :

$$
R^{+}(z) R^{-}(z)=[1-H(z)]^{-1}\left(1-z^{-1}\right)
$$

to obtain

$$
\begin{aligned}
\sum_{i=n}^{\infty} p_{i} z^{i} R^{+}(z)^{-1}= & {\left[\Phi^{-}(1)-\Phi^{-}(z)\right] z(z-1)^{-1} R^{-}(z) } \\
+ & \sum_{i=0}^{n-1} p_{i}\left[A_{i}(z)-z^{i}\right] z(z-1)^{-1} R^{-}(z)
\end{aligned}
$$

Apply $T^{0}$ to both sides. The left side will not change, as it is analytic in $\Gamma^{+}$and continuous on $\Gamma$. On the right side the effect of $T^{0}$ on the first part of it will be some constant $c$, as the first part is obviously analytic in $\Gamma^{-}$. So we obtain

$$
\sum_{i=n}^{\infty} p_{i} z^{i} R^{+}(z)^{-1}=c+T^{0} \sum_{i=0}^{n-1} p_{i}\left[A_{i}(z)-z^{i}\right] z(z-1)^{-1} R^{-}(z) .
$$

The system of equations (32) is the obvious result of application of $D^{j}$ to (39); (33) follows from (39) and $P(1)=1$.

Now (30) readily follows from (39) on the strength of (32) with respect to (21) and the fact that $T^{+} z^{1-n}(z-1)^{-1}\left(1-z^{i}\right) R^{-}(z)=0, i<n$.

The steady state probabilities $p_{i}, i=0,1, \ldots, n-1$, and $c$ constitute one solution of the system (32) and (33). Suppose there is another: 
$\hat{p}_{i}$ and $\hat{c}$. Then using (30) we obtain a $\hat{P}(z)$ that obviously satisfies (36) (with respect to (35)) and hence the vector $\hat{\mathbf{P}}$ of its Taylor expansion coefficients satisfies (34). By construction, $\hat{P}(z) \in L^{1}$, so $\hat{\mathbf{P}}$ has absolutely summable elements, the sum of which is 1 due to (33). According to Chung [6], there can be only one such solution of (34), so $\hat{\mathbf{P}}=\mathbf{P}$. Hence the system (32) and (33) has a unique solution.

The proof of Theorem 4 is over.

For $n=0$ we can obtain $P(z)$ directly from (39). After normalizing we have

$$
P(z)=R^{+}(1)^{-1} R^{+}(z) .
$$

This formula completes our investigation of state probabilities for Markov chains with quasitoeplitz transition matrix of the general type. In articles to follow we investigate the first-zero-hitting problem for these chains and then specify our general results for some important particular cases encountered in applications.

\section{References}

[1] Abolnikov, L.M., Dukhovny, A.M. (1980). Markov Chains with $\Delta_{m, n}$ $\left(\Delta_{m, n}^{\prime}\right)$ Transition Matrix and their Applications to the Queueing Systems with Bulk Arrival, Batch Service and Feedback. Izvestia ANSSSR, Teknicheskaya Kibernetika,1:112-113.

[2] Abolnikov, L.M., Dukhovny, A.M. (1987). Necessary and Sufficient Conditions for the Ergodicity of Markov Chains with Transition $\Delta_{m, n}$ $\left(\Delta_{m, n}^{\prime}\right)$ Matrix. Journal of Applied Mathematics and Simulation, 1:1324.

[3] Dukhovny, A.M. (1981). An Application of Integral Equations in the Analysis of Markov Chains. Teor. Verojatnost. i Primenen., 3:650-651.

[4] Foster, F.G. (1953). On the Stochastic Matrices Associated with Certain Queueing Processes. Ann. Math. Statistics, 24: 355-360.

[5] Gakhov, F.D. Boundary Value Problems. Fizmatgiz, Moscow, 1977.

[6] Kai-lai Chung. Markov Chains with Stationary Transition Probabilities. Springer-Verlag, New York, 1967.

[7] Neuts, M.F. (1979). Queues Solvable without Rouche's Theorem. Oper. Res., 27(4):767-781. 


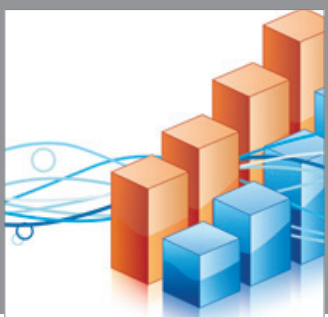

Advances in

Operations Research

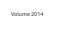

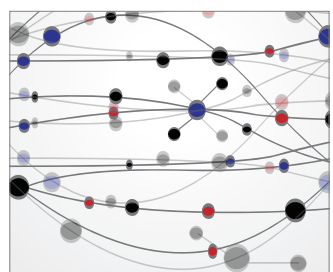

\section{The Scientific} World Journal
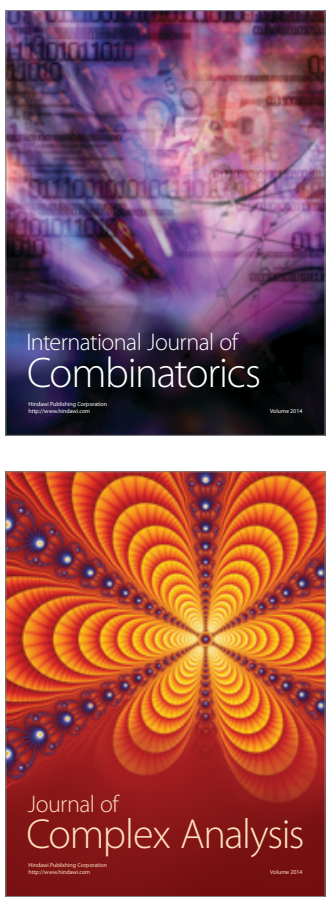

International Journal of

Mathematics and

Mathematical

Sciences
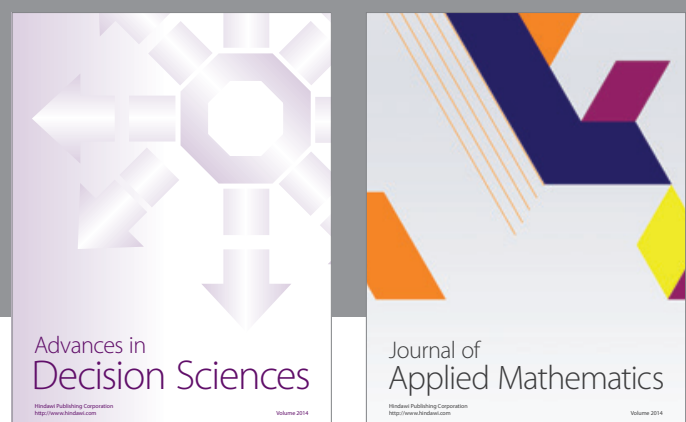

Journal of

Applied Mathematics
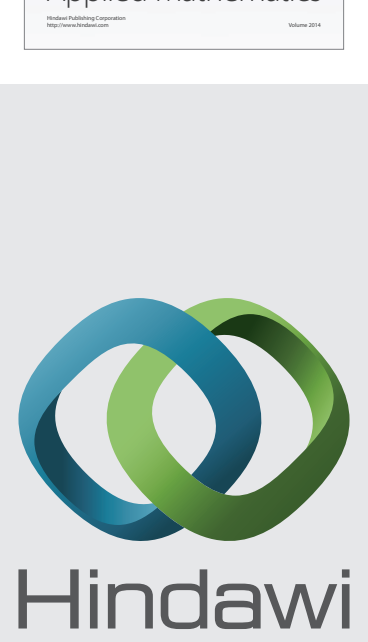

Submit your manuscripts at http://www.hindawi.com
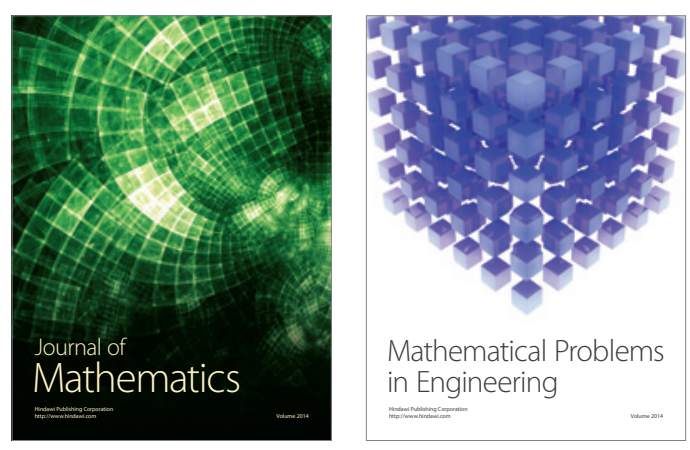

Mathematical Problems in Engineering
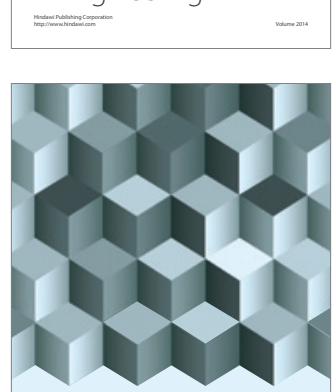

Journal of

Function Spaces
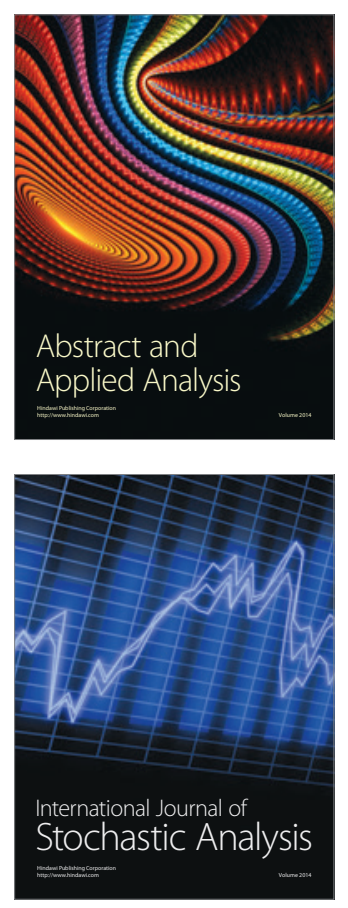

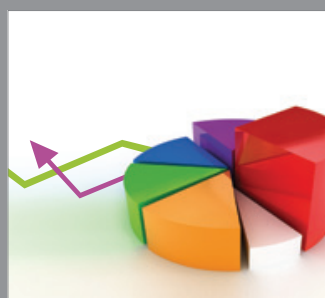

ournal of

Probability and Statistics

Promensencen
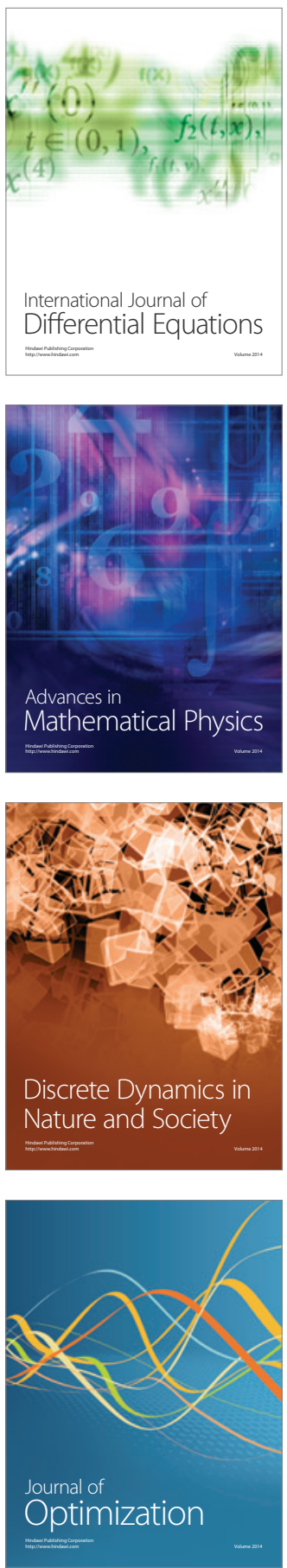